\title{
PREVALENCIA DE ANEMIA Y FACTORES ASOCIADOS EN ADULTOS MAYORES PERUANOS
}

\author{
Carolina Tarqui-Mamani ${ }^{1,2, a}$, José Sanchez-Abanto1,b ${ }^{1,}$ Doris Alvarez-Dongo ${ }^{1, c}$, Paula Espinoza-Oriundo ${ }^{1, c}$, \\ Teresa Jordan-Lechuga ${ }^{1 d}$
}

\begin{abstract}
RESUMEN
Objetivos. Determinar la prevalencia de anemia y factores asociados en los adultos mayores del Perú. Materiales y métodos. Se realizó un estudio transversal durante el año 2011. El muestreo fue probabilístico, estratificado y multietápico. La muestra de viviendas fue 5792 y se incluyó 2172 adultos mayores. Se definió anemia como hemoglobina <13,0 g/ $\mathrm{dL}$ en hombres y $<12,0 \mathrm{~g} / \mathrm{dL}$ en mujeres. El estado nutricional se evaluó mediante el IMC clasificándose como delgadez (IMC $\leq 23,0)$, normal (IMC>23 a <28), sobrepeso (IMC $\geq 28$ a $<32,0)$ y obesidad (IMC $\geq 32$ ). El análisis estadístico se realizó por muestras complejas y se ajustó por factor de ponderación. Se calcularon las medias, proporciones, chi cuadrado y regresión logística. Resultados. El promedio de hemoglobina fue $13,4 \pm 1,6 \mathrm{~g} / \mathrm{dL}$. La prevalencia de anemia fue 23,3\% (Leve: 17,1\%; moderada: 5,7\% y severa: 0,5\%). La edad de 70 a 79 años (OR 1,5; IC 95\%: 1,1-2,0), >80 años (OR 2,1; IC 95\%: 1,4-3,0) y la delgadez (OR 1,7; IC 95\%:1,2-2,3) se asociaron con la anemia. Los departamentos con mayor prevalencia de anemia fueron Ayacucho (57,6\%), Ancash (40,1\%), Lambayeque (37,7\%) y Apurímac (36,9\%). Conclusiones. Aproximadamente la cuarta parte de los adultos mayores tuvieron anemia, siendo más predominante en los analfabetos, procedentes de áreas rurales y pobres. La mayor edad y la delgadez se asocian con la presencia de anemia en los adultos mayores peruanos.
\end{abstract}

Palabras clave: Adulto mayor; Anemia; Delgadez; Sobrepeso; Obesidad (fuente: DeCS BIREME)

\section{PREVALENCE OF ANEMIA AND ASSOCIATED FACTORS IN ELDERLY RESIDING IN PERUVIAN HOUSEHOLDS}

\begin{abstract}
Objectives. To estimate the prevalence of anemia and associated factors in elderly residing in Peruvian households. Materials and methods. the study deals with a cross-sectional design and was conducted in 2011. The sample was probabilistic, stratified and multistage independent in department of Peru. The required sample housing was 5792, we included 2172 elderly. We asked informed consent of all elderly. The anemia was defined as hemoglobin $<13.0 \mathrm{~g} / \mathrm{dL}$ in men and $<12.0 \mathrm{~g} / \mathrm{dL}$ in women, hemoglobin was adjusted for altitude. The anthropometric measurements were performed according to methodology MINSA/INS. The Nutritional status was assessed by body mass index. The classification of nutritional status: underweight $(B M I \leq 23,0)(B M I>23$ to $<28)$, overweight $(B M I \geq 28$ to $<32.0)$ and obesity $(B M I \geq 32)$. Statistical analysis was performed using complex samples and adjusted by the weighting factor. We Calculated means, proportions. The chi-square and regression logistic. Results. The mean hemoglobin was $13.4 \pm 1.6 \mathrm{~g} / \mathrm{dL}$. The prevalence of anemia was 23,3\% (mild anemia $17.1 \%$, moderate: severe $5,7 \%$ and $0,5 \%$ ). The age 70 to 79 years (OR 1.5; Cl 95\%:1.1; 2.0), > 80 years (OR 2.1; Cl 95\%: 1.4; 3.0) and thinness (OR 1.7; Cl 95\%: 1.2, 2.3) associated with anemia. Ayacucho, Ancash, Lambayeque and Apurimac were the departments with the highest prevalence of anemia. Conclusions. Approximately one quarter of elderly were anemic, being more prevalent in the illiterate, rural and poor. Older age and thinness are associated with anemia in elderly Peruvians.
\end{abstract}

Key words: Elderly; Anemia; Thinness; Overweight; Obesity (source: MeSH, NLM)

\section{INTRODUCCIÓN}

La anemia es un problema de salud muy frecuente en los adultos mayores, se incrementa a partir de los 50 años (1). La prevalencia de anemia en los adultos mayores de 65 años asciende a $10 \%$ en las mujeres y $11 \%$ en los hombres y aumenta $26,1 \%$ en hombres y $20,1 \%$ en mujeres en los adultos mayores de 85 años ${ }^{(2)}$. Algunos estudios muestran que la prevalencia de anemia en los adultos mayores es variada y fluctúa entre 2,9 a $61 \%$ en los varones y 3,3 a $41,0 \%$ en las mujeres ${ }^{(3)}$. En el Perú, estudios realizados en adultos mayores hospitalizados muestran una frecuencia de anemia de $42^{(4)}$ y $76,4 \%{ }^{(5)}$.

\footnotetext{
Centro Nacional de Alimentación y Nutrición, Instituto Nacional de Salud. Lima, Perú

Departamento de Medicina Preventiva y Salud Pública, Facultad de Medicina Humana, Universidad Nacional Mayor de San Marcos. Lima, Perú.

Obstetra, doctora en salud pública, magíster en epidemiología, especialidad en estadística; ${ }^{\mathrm{b}}$ nutricionista, magíster en nutrición; ${ }^{\mathrm{c}}$ nutricionista; ${ }^{\mathrm{d}}$ bióloga. Recibido: 12-09-14 Aprobado: 22-04-15
}

Citar como: Tarqui-Mamani C, Sanchez-Abanto J, Alvarez-Dongo D, Espinoza-Oriundo P, Jordan-Lechuga T. Prevalencia de anemia y factores asociados en adultos mayores peruanos. Rev Peru Med Exp Salud Publica. 2015;32(4):687-92. 
La anemia se incrementa con la edad (6,7) y está asociada con cambios en los estilos de vida, además de incrementar el riesgo por caídas, producir infecciones, disminuir el estado cognoscitivo y la capacidad funcional ${ }^{(8)}$. Con frecuencia la anemia tiende a ser subdiagnosticada debido a la coexistencia con alguna enfermedad ${ }^{(9)}$. Las causas de la anemia en los adultos mayores pueden ser clasificadas en tres categorías: deficiencia de la ingesta de hierro en la dieta, anemia asociada a enfermedades renales crónicas y la anemia no explicada ${ }^{(10)}$. Esta última se atribuye a la disminución de los niveles de hemoglobina, posiblemente por la respuesta eritropoyética en la deficiencia de hierro; altos niveles de citoquinas proinflamatorias; la disminución de los niveles de andrógenos, y disminución proliferativa y regenerativa de las células de la médula ósea, mielodisplasia que está asociada con la disminución de la expectativa de vida (11). La anemia en adultos mayores aumenta la mortalidad y morbimortalidad cardiovascular, y está asociada con mayor deterioro cognoscitivo, fragilidad, disminución de la calidad de vida, mayor riesgo de lesiones en el hogar, entre otros ${ }^{(12,13)}$.

Se han realizado diversos estudios en adultos mayores hospitalizados, las que se caracterizan por emplear muestras pequeñas no probabilísticas. Esta es la primera vez que se estima la prevalencia nacional de anemia en los adultos mayores mediante un estudio poblacional con inferencia nacional, lo que permitirá tener un panorama real de la anemia como problema de salud pública en los adultos mayores peruanos.

El objetivo del estudio fue estimar la prevalencia de anemia y factores asociados en los adultos mayores que residen en los hogares peruanos.

\section{MATERIALES Y MÉTODOS}

\section{DISEÑO Y POBLACIÒN DE ESTUDIO}

Se realizó un estudio observacional y transversal. Se incluyó a los adultos mayores, residentes en los hogares peruanos, que voluntariamente aceptaron participar en el estudio y se excluyó a los adultos mayores con limitaciones físicas (malformaciones congénitas, escoliosis acentuada que imposibilite la aplicación de la técnica antropométrica) que dificulten las mediciones antropométricas. El recojo de datos se realizó durante el 2011. El muestreo fue probabilístico, estratificado y multietápico e independiente en cada departamento del Perú. El tamaño de la muestra se calculó para estimar características de la vivienda y del hogar, características de los miembros del hogar, educación, salud, empleo, ingreso y gastos del hogar. Se consideró: nivel de confianza al 95\%, precisión $5 \%$, total de viviendas en el Perú, la tasa de no respuesta y el efecto de diseño correspondiente a los reportes previos de la Encuesta Nacional de Hogares (ENAHO); 2011. La muestra requerida fue 5792 viviendas (3384 en la zona urbana y 2408 en la rural). Se seleccionaron aleatoriamente seis viviendas en cada conglomerado urbano y ocho viviendas en el rural (14). El estudio se realizó en el marco del convenio entre Instituto Nacional de Estadística e Informática (INEI) y el Instituto Nacional de Salud (INS), mediante el cual, el INEI provee la muestra y cartografías, y el INS a través del Centro Nacional de Alimentación y Nutrición (CENAN) realizó la evaluación antropométrica y bioquímica en la población comprendida en la muestra. Se incluyó 2172 adultos mayores (60 años a más) a nivel nacional.

\section{TÉCNICAS E INSTRUMENTOS}

La anemia fue definida como la concentración de hemoglobina $(\mathrm{Hb})<13,0 \mathrm{~g} / \mathrm{dL}$ en hombres $\mathrm{y}<12,0 \mathrm{~g} /$ $\mathrm{dL}$ en mujeres. Se consideró anemia leve $(\mathrm{Hb} \geq 12,0 \mathrm{~g} /$ $\mathrm{dL}$ a $\leq 12,9 \mathrm{~g} / \mathrm{dL}$ ), moderada ( $\mathrm{Hb} \geq 9,0 \mathrm{~g} / \mathrm{d}$ a $\leq 11,9 \mathrm{~g} /$ $\mathrm{dL})$ y severa $(\mathrm{Hb}<9,0 \mathrm{~g} / \mathrm{dL})$ en hombres. También se consideró anemia leve $(\mathrm{Hb} \geq 10,0 \mathrm{~g} / \mathrm{dL}$ a $\leq 11,9 \mathrm{~g} / \mathrm{dL})$, moderada $(\mathrm{Hb} \geq 7,0 \mathrm{~g} / \mathrm{dL} a \leq 9,9 \mathrm{~g} / \mathrm{dL})$ y severa $(\mathrm{Hb}<7,0 \mathrm{~g} /$ $\mathrm{dL}$ ) en mujeres ${ }^{(15)}$. Las concentraciones de hemoglobina se cuantificaron en muestras de sangre capilar con un hemoglobinómetro portátil. La hemoglobina se ajustó por la altitud sobre el nivel del mar, según la siguiente fórmula: Ajuste $=0,022^{*}\left((\text { altitud } / 1000)^{*} 3,3^{*}(\text { altitud } / 1000)^{*} 3,3\right)$ $\left(0,032^{*}\left((\text { altitud } / 1000)^{*} 3,3\right)\right)$.

Las mediciones antropométricas se realizaron según metodología del MINSA/INS, se utilizó balanzas digitales calibradas con capacidad de $150 \mathrm{~kg}$ y precisión de $\pm 100 \mathrm{~g}$ y tallímetros móviles de madera. El personal que realizó la medición de talla y peso fue capacitado y estandarizado por el personal del Centro Nacional de Alimentación y Nutrición del INS. El estado nutricional se evalúo mediante el índice de masa corporal (IMC) según la clasificación del estado nutricional para el adulto mayor del Ministerio de Salud, delgadez $(I M C \leq 23,0)$, normal (IMC>23 a $<28$ ), sobrepeso (IMC $\geq 28$ a $<32,0)$ y obesidad (IMC $\geq 32)^{(16)}$.

La pobreza se midió por enfoque monetario (hogar pobre, hogar pobre extremo y no pobre), se consideró: "hogar pobre" a los hogares que solamente cubren la alimentación, pero no alcanzan a cubrir otras necesidades como bienes y servicios, entre ellos educación, salud, calzado, transporte, o vivienda. "Hogar pobre extremo", a los hogares que no pueden 
cubrir su gastos en alimentos ni sus gastos en otros bienes y servicios como transporte, vestimenta, vivienda, educación y salud. Finalmente "hogar no pobre", cuando el hogar cubre la canasta familiar que satisface las necesidades nutricionales de las personas y otras necesidades como transporte, vestimenta, vivienda, educación y salud ${ }^{(17)}$.

\section{ANÁLISIS DE LOS DATOS}

El procesamiento se realizó mediante el programa SPSS v22 para muestras complejas y se ajustó por factor de ponderación. Se calculó las medias, proporciones con intervalo de confianza al $95 \%$. Se calculó el chi cuadrado y la regresión logística para evaluar la asociación entre las covariables y la anemia.

\section{CONSIDERACIONES ÉTICAS}

Se solicitó el consentimiento informado escrito a todos los participantes y se acreditó mediante la firma o huella digital en el caso de los analfabetos, este procedimiento se realizó bajo la presencia de un testigo. El estudio se realizó en el marco de la Vigilancia de Indicadores Nutricionales que realiza la Dirección Ejecutiva de Vigilancia Alimentaria y Nutricional del Instituto Nacional de Salud en convenio con el Instituto Nacional de Estadística e Informática y fue registrado y aprobado en el Plan Operativo Institucional del Instituto Nacional de Salud, por ello, no fue evaluado por el Comité de Ética del INS.

\section{RESULTADOS}

Se incluyó 2172 adultos mayores, 60,6\% fueron convivientes o casados, el $77,7 \%$ tuvieron nivel educativo primaria, secundaria o superior, la mayoría tuvo algún tipo de seguro de salud y el $21,3 \%$ fueron pobres (pobreza extrema o pobreza) (Tabla 1).

El promedio de hemoglobina fue 13,4 $\pm 1,6 \mathrm{~g} / \mathrm{dL}$, siendo mayor en los varones $13,8 \pm 1,8 \mathrm{~g} / \mathrm{dL}$ que en las mujeres $12,9 \pm 1,4 \mathrm{~g} / \mathrm{dL}$. El promedio de hemoglobina fue mayor en los obesos (14,1 g/dL), seguido del sobrepeso $(13,6$ $\mathrm{g} / \mathrm{dL})$ y normal $(13,4 \mathrm{~g} / \mathrm{dL})$ y más bajo en la delgadez $(12,9 \mathrm{~g} / \mathrm{dL})$. La prevalencia de anemia fue $23,3 \%(21,1 \%$; $25,7 \%)$, de los cuales, $17,1 \%(15,2 \% ; 19,3 \%)$ anemia leve, $5,7 \%(4,6 \% ; 7,0 \%)$ moderada y $0,5 \%(0,2 \% ; 1,1 \%)$ severa (Tabla 2 ).

Los departamentos con mayor prevalencia de anemia fueron Ayacucho (57,6\%), Ancash (40,1\%), Lambayeque $(37,7 \%)$ y Apurímac (36,9\%). Cajamarca $(9,5 \%)$ y Arequipa $(8,8 \%)$ tuvieron las prevalencias de anemia más bajas a nivel nacional (Figura 1).
Tabla 1. Características de los adultos mayores peruanos, 2011

\begin{tabular}{|c|c|c|c|}
\hline Características & $\mathbf{n}$ & $\%$ & (IC 95\%) \\
\hline \multicolumn{4}{|l|}{ Edad } \\
\hline $60-69$ & 1060 & 47,6 & $(44,4-50,8)$ \\
\hline $70-79$ & 754 & 35,5 & $(32,7-38,4)$ \\
\hline$>80$ & 358 & 16,9 & $(14,8-19,2)$ \\
\hline \multicolumn{4}{|l|}{ Sexo } \\
\hline Hombre & 1040 & 49,1 & $(46,9-51,4)$ \\
\hline Mujer & 1132 & 50,9 & $(48,6-53,1)$ \\
\hline \multicolumn{4}{|l|}{ Estado civil } \\
\hline Conviviente & 221 & 9,6 & $(7,7-12,1)$ \\
\hline Casado & 1134 & 51,0 & $(47,9-54,1)$ \\
\hline Viudo & 719 & 34,4 & $(31,7-37,2)$ \\
\hline Divorciado/Separado/Soltero & 98 & 5,0 & $(3,7-6,6)$ \\
\hline \multicolumn{4}{|l|}{ Nivel educativo } \\
\hline Analfabeto & 592 & 22,3 & $(20,1-24,7)$ \\
\hline Primaria & 1077 & 46,3 & $(43,1-49,6)$ \\
\hline Secundaria & 345 & 20,5 & $(18,1-23,1)$ \\
\hline Superior & 157 & 10,9 & $(8,4-14,1)$ \\
\hline \multicolumn{4}{|l|}{ Seguro de salud } \\
\hline No & 635 & 30,3 & $(27,2-33,6)$ \\
\hline Sí & 1536 & 69,7 & $(66,4-72,8)$ \\
\hline \multicolumn{4}{|l|}{ Área } \\
\hline Urbano & 1198 & 70,8 & $(67,3-74,2)$ \\
\hline Rural & 974 & 29,2 & $(25,8-32,7)$ \\
\hline \multicolumn{4}{|l|}{ Dominio geográfico } \\
\hline Lima Metropolitana & 235 & 30,8 & $(27,1-34,7)$ \\
\hline Costa & 643 & 24,3 & $(21,6-27,3)$ \\
\hline Sierra & 900 & 33,6 & $(30,5-36,8)$ \\
\hline Selva & 394 & 11,3 & $(9,6-13,3)$ \\
\hline \multicolumn{4}{|l|}{ Nivel de pobreza } \\
\hline Pobre extremo & 193 & 6,1 & $(4,9-7,5)$ \\
\hline Pobre no extremo & 407 & 15,2 & $(13,0-17,7)$ \\
\hline No pobre & 1572 & 78,7 & $(75,8-81,3)$ \\
\hline
\end{tabular}

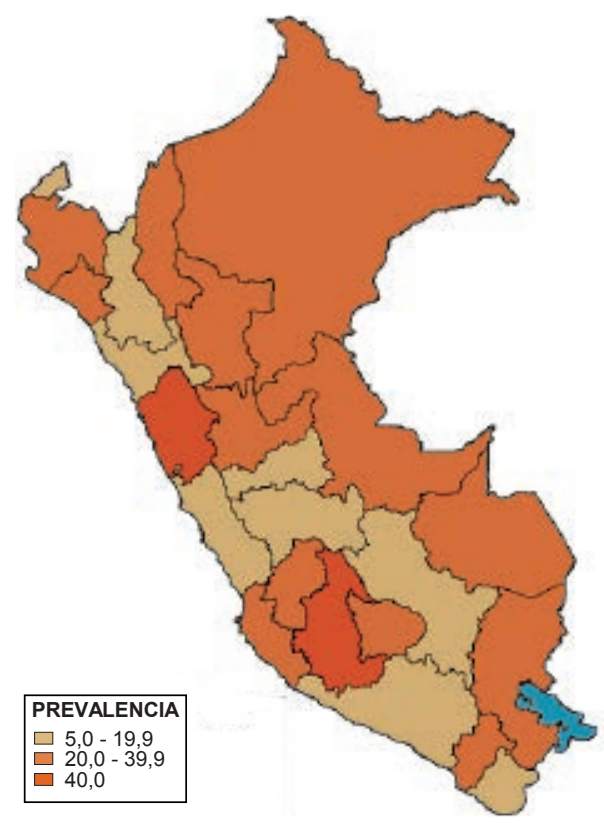

Figura 1. Distribución de la anemia en los adultos mayores. Perú; 2011 
Tabla 2. Características de los adultos mayores peruanos según condición de anemia; 2011

\begin{tabular}{|c|c|c|c|c|c|c|c|}
\hline \multirow{2}{*}{ Variables } & \multicolumn{2}{|c|}{ Con anemia } & \multicolumn{2}{|c|}{ Sin anemia } & \multirow{2}{*}{ OR } & \multirow{2}{*}{ (IC 95\%) } & \multirow{2}{*}{ Valor $p$} \\
\hline & $\mathrm{n}$ & $(\%)$ & $\mathbf{n}$ & $(\%)$ & & & \\
\hline \multicolumn{8}{|l|}{ Edad } \\
\hline $60-69$ & 193 & $(17,0)$ & 862 & $(83,0)$ & 1,0 & & $<0,001$ \\
\hline $70-79$ & 217 & $(25,9)$ & 537 & $(74,1)$ & 1,5 & $(1,1-2,0)$ & \\
\hline$>80$ & 141 & $(35,7)$ & 216 & $(64,3)$ & 2,1 & $(1,4-3,0)$ & \\
\hline \multicolumn{8}{|l|}{ Sexo } \\
\hline Hombre & 277 & $(25,5)$ & 758 & $(74,5)$ & 1,0 & & 0,048 \\
\hline Mujer & 274 & $(21,2)$ & 857 & $(78,8)$ & 0,8 & $(0,6-1,0)$ & \\
\hline \multicolumn{8}{|l|}{ Pareja } \\
\hline Sí & 341 & $(23,4)$ & 1008 & $(76,6)$ & 1,0 & & 0,885 \\
\hline No & 210 & $(23,1)$ & 607 & $(76,9)$ & 0,9 & $(0,7-1,2)$ & \\
\hline \multicolumn{8}{|l|}{ Nivel educativo } \\
\hline Superior & 18 & $(12,9)$ & 138 & $(87,1)$ & 1,0 & & 0,002 \\
\hline Secundaria & 55 & $(17,0)$ & 287 & $(83,0)$ & 1,3 & $(0,6-2,7)$ & \\
\hline Primaria & 304 & $(26,4)$ & 771 & $(73,6)$ & 2,0 & $(0,9-4,1)$ & \\
\hline Analfabeto & 174 & $(27,9)$ & 418 & $(72,1)$ & 1,9 & $(0,9-4,0)$ & \\
\hline \multicolumn{8}{|l|}{ Seguro de salud } \\
\hline Sí & 391 & $(23,2)$ & 1141 & $(76,8)$ & 1,0 & & 0,886 \\
\hline No & 160 & $(23,6)$ & 473 & $(76,4)$ & 1,0 & $(0,7-1,4)$ & \\
\hline \multicolumn{8}{|l|}{ Estado nutricional } \\
\hline Normal & 209 & $(22,3)$ & 658 & $(77,7)$ & 1,0 & & $<0,001$ \\
\hline Delgadez & 240 & $(35,0)$ & 431 & $(65,0)$ & 1,7 & $(1,2-2,3)$ & \\
\hline Sobrepeso & 53 & $(13,5)$ & 328 & $(86,5)$ & 0,6 & $(0,4-1,0)$ & \\
\hline Obesidad & 19 & $(10,1)$ & 151 & $(89,9)$ & 0,4 & $(0,3-0,8)$ & \\
\hline \multicolumn{8}{|l|}{ Área } \\
\hline Urbano & 274 & $(20,9)$ & 918 & $(79,1)$ & 1,0 & & 0,001 \\
\hline Rural & 277 & $(29,2)$ & 697 & $(70,8)$ & 1,1 & $(0,8-1,5)$ & \\
\hline \multicolumn{8}{|l|}{ Dominio geográfico } \\
\hline Lima Metropolitana & 49 & $(18,7)$ & 186 & $(81,3)$ & 1,0 & & 0,057 \\
\hline Costa & 170 & $(26,9)$ & 473 & $(73,1)$ & 1,5 & $(1,0-2,4)$ & \\
\hline Sierra & 239 & $(24,8)$ & 661 & $(75,2)$ & 0,9 & $(0,6-1,5)$ & \\
\hline Selva & 93 & $(23,9)$ & 295 & $(76,1)$ & 1,0 & $(0,6-1,6)$ & \\
\hline \multicolumn{8}{|l|}{ Nivel de pobreza } \\
\hline No pobre & 351 & $(21,2)$ & 1215 & $(78,8)$ & 1,0 & & $<0,001$ \\
\hline Pobre no extremo & 128 & $(29,7)$ & 279 & $(70,3)$ & 1,2 & $(0,9-1,7)$ & \\
\hline Pobre extremo & 72 & $(35,2)$ & 121 & $(64,8)$ & 1,3 & $(0,8-1,9)$ & \\
\hline
\end{tabular}

\section{DISCUSIÓN}

El envejecimiento de la población a nivel mundial avanza progresivamente ${ }^{(18)}$; tanto en los países desarrollados como en vías de desarrollo, sin embargo, la preparación o forma como se afronta este periodo difiere entre los adultos mayores si residen en uno u otro lugar. La mayoría de estudios publicados en adultos mayores fueron realizados en los ambientes hospitalarios. Son muy escasos los estudios de anemia en adultos mayores con base poblacional.

Aproximadamente uno de cada cuatro adultos mayores padece de anemia, nuestros resultados fueron menores a lo reportado por Reyes (42\%) y Ortiz et al. (76.4\%), en Perú ${ }^{(4,5)}$, mucho menor a lo reportado en Chile $(40,1 \%)^{(19)}$ y Nueva Zelandia $(33,3 \%)^{(20)}$, pero mayor que lo reportado por Yavuz et al. (10,3\%) en Turquía (21), todos estos estudios realizados en adultos mayores hospitalizados, y en adultos mayores urbanos de Corea ${ }^{(22)}$.
Una probable explicación para esta diferencia, podría ser que los estudios publicados, en su mayoría, se centran en estimar la prevalencia de anemia en adultos mayores hospitalizados mientras que nuestros resultados tienen base poblacional, lo que permite estimar la prevalencia de anemia en los adultos mayores a nivel nacional, disminuyendo el subregistro del diagnóstico que se realiza en los ambientes hospitalarios donde muchas veces tiende a subdiagnosticar la anemia porque se reporta la enfermedad de fondo. Aunque el estudio no precisa el tipo de anemia, se conoce que la anemia ferropénica es la más frecuente en los adultos mayores ${ }^{(23)}$, y está asociado a las pérdidas microscópicas diarias que disminuyen los depósitos de hierro en el organismo, esta puede ser de origen nasal o digestivo (epistaxis, várices esofágicas, hernia de hiato, divertículos intestinales, malformaciones vasculares, hemorroides, etc.), pérdidas micro o macroscópicas por el tracto urinario (tumores benignos o malignos), las pérdidas de sangre de origen pulmonar, el uso incontrolado de antiinflamatorios no esteroideos 
(AINES) que produce pérdida crónica por sangrado gastrointestinal por gastritis secundaria ${ }^{(24)}$.

Nuestros resultados muestran que a medida que aumenta la edad también aumenta la prevalencia de anemia, similar tendencia se observa en los adultos mayores coreanos, aunque los resultados encontrados son mucho menores y se trata solamente de población urbana ${ }^{(25)}$. También hallamos que la edad está asociada a la condición de anemia, los adultos mayores de 80 años tienen 1,4 veces más probabilidad de tener anemia que los adultos mayores entre 60 a 69 años, y los adultos mayores entre 70 a 79 años tienen 1,5 más probabilidad de tener anemia que los adultos mayores entre 60 a 69 años. Situación que resulta preocupante porque la anemia se relaciona con el deterioro de las funciones físicas y cognoscitivas del adulto mayor; constituyéndose en un paso intermedio hacia las enfermedades crónicas y un predictor de mortalidad debido a la correlación que existe entre la severidad de la anemia y el riesgo de muerte ${ }^{(11,25)}$.

Uno de cada cuatro varones y una de cada cinco mujeres presentó anemia, nuestro análisis demostró asociación entre el sexo masculino y la anemia, aunque el análisis multivariado no mostró asociación significativa, estos resultados difieren de lo reportado por Bang et al. (22), que refieren que las mujeres presentan mayor prevalencia de anemia que los varones.

El nivel educativo no estuvo asociado a la anemia, pese a la influencia que tiene el conocimiento sobre los alimentos ricos en hierro o alimentos que favorecen o inhiben la absorción de hierro; por otro lado, la probable mejor capacidad adquisitiva debido a la condición de jubilado y contar con algún tipo de seguro de salud que favorecería la atención y tratamiento oportuno de las enfermedades crónicas que padece el adulto mayor.

Uno de cada diez adultos mayores con obesidad presentó anemia, y uno de cada tres adultos mayores con delgadez presento anemia, este hallazgo es similar a lo reportado por Samper-Ternent et al. ${ }^{(26)}$, que describe que el $10 \%$ de adultos mayores mexicanos presentó obesidad y anemia.

Por otra parte, se observó una tendencia porcentual ascendente entre el nivel de pobreza y la anemia. Resulta preocupante que uno de cada tres pobres extremos, y dos de cada seis pobres no extremos presenten anemia. Situación que podría estar relacionada con la baja capacidad adquisitiva que tienen los hogares pobres para adquirir alimentos fuentes de hierro y suplementos como el sulfato ferroso y multimicronutrientes. Por ello, sería conveniente ampliar el estudio para especificar las etiologías carenciales, la presencia de alteraciones en la absorción de hierro debido a la presencia de parásitos que frecuentemente son altas en las zonas de pobreza y extrema pobreza.

Los departamentos con mayor prevalencia de anemia corresponden a los departamentos que aún se consideran que tienen pobreza extrema ${ }^{(17)}$. Al respecto, se sabe que la pobreza es un determinante social de la salud, en este sentido, también podría influir en la deficiente ingesta de alimentos fuente de hierro en la dieta, acceso a la atención de salud y el posible abandono de los adultos mayores. Por otra parte, los departamentos con prevalencias de anemia más bajas a nivel nacional, se caracterizan porque tienen como actividad principal la minería, la ganadería y la agricultura, es posible que dicha situación contribuya a mejorar la disponibilidad alimentaria, acceso y consumo de alimentos fuentes de hierro.

Una limitación del estudio fue que se realizó a nivel de los hogares peruanos, por ende los resultados del estudio podrían estar subestimando la prevalencia real de anemia debido a que no refleja la situación de los adultos mayores que están hospitalizados, sin embargo, considerando el tamaño y la representatividad de la muestra, podría brindar una primera aproximación de la prevalencia de anemia en los adultos mayores peruanos, por otro lado, el estudio no precisa, la enfermedad de fondo ni el tipo de anemia, al respecto, según Reyes (4) el tipo de anemia más frecuente en adultos mayores peruanos fue por enfermedad crónica, seguido por el tipo megaloblástico, y por último el ferropénico.

En conclusión, la cuarta parte de los adultos mayores tuvieron anemia, siendo mayor en los analfabetos, los que procedían de áreas rurales y pobres extremos o pobres. Existe una tendencia porcentual ascendente conforme aumenta la edad en los adultos mayores. La mayor edad y la delgadez se asocian con la anemia en los adultos mayores peruanos. Se sugiere a las autoridades sanitarias, la suplementación con hierro o multivitamínicos en los adultos mayores, sobre todo en las zonas rurales, en condición de pobreza y situación de abandono. Asimismo, el tratamiento especializado en los casos de los adultos mayores con malnutrición (delgadez o exceso) y anemia debido al incremento de riesgo de mortalidad. También, se sugiere ampliar la cobertura del Seguro Integral de Salud a todos los adultos mayores a fin de mejorar el acceso al servicio de salud y la implementación de albergues sobre todo para los adultos mayores que residen en la zonas rurales o pobres, que podrían estar en condición de abandono, a fin de mejorar la calidad de vida de este grupo en situación de vulnerabilidad.

Agradecimientos: al equipo técnico de supervisión, bioquímica y antropometría de la Dirección Ejecutiva de Vigilancia Alimentaria y Nutricional del Centro Nacional de Alimentación y Nutrición del INS. 
Contribuciones de autoría: CTM ha participado en la concepción del artículo, procesamiento de datos, análisis, redacción y aprobación de la versión final. JSA participó en la redacción y aprobó la versión final. DAD participó en la redacción y aprobó la versión final. PLEO participó en la redacción y aprobó la versión final del artículo y TJL participó en la redacción y aprobó la versión final.
Fuentes de financiamiento: este estudio fue financiado por el Instituto Nacional de Salud y se realizó en el marco del convenio suscrito entre el Instituto Nacional de Estadística e Informática y el Instituto Nacional de Salud.

Conflictos de interés: los autores declaran no tener conflictos de interés.

\section{REFERENCIAS BIBLIOGRÁFICAS}

1. Ania BJ, Suman VJ, Fairbanks VF Rademacher DM, Melton LJ 3rd. Incidence of anemia in older people: an epidemiologic study in a well defined population. J Am Geriatr Soc. $1997 ; 45(7): 825-831$

2. Guralnik JM, Eisenstaedt RS, Ferrucci L, Klein HG, Woodman RC. Prevalence of anemia in persons 65 years and older in the United States: evidence for a high rate of unexplained anemia. Blood. 2004;104(8):2263-8.

3. Beghe C, Wilson A, Ershler W. Prevalence and outcomes of anemia in geriatrics a systematic review of the literature. Am J Med. 2004;116 Suppl 7A:3S-10S.

4. Reyes E. Prevalencia de anemia en el anciano hospitalizado en el hospital Central de la Fuerza Aérea en los servicios de Medicina y Geriatría en el periodo de enero a marzo 2002 [Tesis para obtener especialidad de medicina interna] Lima: Facultad de Medicina Humana, UNMSM; 2002.

5. Ortiz P, Mendez F, Varela L, Pamo O. Variación del estado nutricional del paciente adulto mayor durante la hospitalización en los servicios de medicina de un hospital general. Rev Med Hered. 2007;18(1):3-9

6. Gabrilove J. Anemia and the elderly: clinical considerations. Best Pract Res Clin Haematol. 2005; 18(3):417-22.

7. Andres E, Federici L, Serraj K, Kaltenbach G. Update of nutrientdeficiency anemia in elderly patients. Eur J Intern Med. 2008;19(7):488-93. doi: 10.1016/j.ejim.2008.01.016.

8. Lash AA, Coyer SM. Anemia in older adults. Medsurg Nurs 2008; 17(5):298304.

9. Carmel R. Anemia and aging: an overview of clinical, diagnostic and biological issues. Blood Rev. 2001;15(1):9-18

10. Morón C, Viteri FE. Update on common indicators of nutritional status: food access, food consumption, and biochemical measures of iron and anemia. Nutr Rev. 2009;67 Suppl 1:S31-5. doi: 10.1111/j.17534887.2009.00156.x.

11. Paltiel O, Clarfield M. Anemia in elderly people: Risk marker or risk factor? CMAJ. 2009;181(3-4):129-30 doi: 10.1503/cmaj.091199.

12. Penninx BW, Pahor M, Cesari M, Corsi AM, Woodman RC, Bandinelli S, et al. Anemia is associated with disability and decreased physical performance and muscle strength in the elderly. J Am Geriatr Soc. 2004;52(5):719-24

13. Cesari M, Penninx BW, Lauretani F, Russo CR, Carter C, Bandinelli S, et al. Hemoglobin levels and skeletal muscle: results from the InCHIANTI Study. J Gerontol A Biol Sci Med Sci. 2004;59(3):249-54.

14. Instituto Nacional de Estadística e Informática. Encuesta Nacional de Hogares. Lima: INEI; 2011.

15. Gleason G, Scrimshaw N. An overview of the functional significance of iron deficiency. In: Kraemer $\mathrm{K}$, Zimmermann MB, editors. Nutritional anemia. Basel (Switzerland): Sight and Life Press; 2007.

16. Instituto Nacional de Salud. Guía técnica para la valoración nutricional antropométrica de la persona adulta mayor. Lima (Perú): INS;2013

17. Instituto Nacional de Estadística e Informática (INEI). Evolución de la pobreza monetaria 2007-2012 [internet]. Lima: INEI; 2013 [Citado el 10 de febrero de 2014]. Disponible en: http://www.inei.gob.pe/media/ cifras_de_pobreza/pobreza_ informetecnico2013_1.pdf

18. Organización de las Naciones Unidas. Segunda Asamblea Mundial Sobre el Envejecimiento. Madrid: ONU; 2002.

19. Lopez-Sierra M, Calderon S, Gomez J, Pilleux L. Prevalence of Anaemia and Evaluation of Transferrin Receptor (sTfR) in the Diagnosis of Iron Deficiency in the Hospitalized Elderly
Patients: Anaemia Clinical Studies in Chile. Anemia. 2012;2012:646201. doi: $10.1155 / 2012 / 646201$

20. Nathavitharana RL, Murray JA, D'Sousa N, Sheehan T, Frampton CM, Baker BW. Anaemia is highly prevalent among unselected internal medicine inpatients and is associated with increased mortality, earlier readmission and more prolonged hospital stay: an observational retrospective cohort study. Intern Med J. 2012;42(6):683-91. doi: 10.1111/j.1445-5994.2011.02566.x.

21. Yavuz BB, Cankurtaran $M$, Haznedaroglu IC, Halil M, Ulger $\mathrm{Z}$, Altun $\mathrm{B}$, et al. Iron deficiency can cause cognitive impairment in geriatric patients. J Nutr Health Aging. 2012;16(3):220-4.

22. Bang SM, Lee JO, Kim YJ, Lee KW, Lim $\mathrm{S}$, Kim JH, et al. Anemia and activities of daily living in the Korean urban elderly population: Results from the Korean Longitudinal Study on Health and Aging (KLoSHA). Ann Hematol. 2013;92(1):59-65. doi: 10.1007/ s00277-012-1563-6.

23. Irles R. Anemias en el anciano y su tratamiento. Investig Clin 2005;8(3):256-60.

24. Wolff MM, Lichtenstein DR, Singh G. Gastrointestinal toxicity of nonsteroidal antiinflamamtory drugs. N Engl J Med. 1999;340(24):1888-99.

25. Duh MS, Mody SH, Lefebvre P, Woodman RC, Buteau S, Piech CT. Anaemia and the risk of injurious falls in a community-dwelling elderly population. Drugs Aging. 2008;25(4): 325-34

26. Samper-Ternent R, Michaels-Obregon A, Wong R. Coexistence of Obesity and Anemia in Older Mexican Adults. Ageing Int. 2011;37(1):104-117.

Correspondencia: Carolina Tarqui-Mamani Dirección: Calle Tizón y Bueno 276; Lima 11, Perú

Correo electrónico:carobtm@gmail.com 